$\overline{\text { Report }}$

\title{
Assessment of Three Methods for the Identification of Enzymatically Hydrolyzed Guar Gum
}

\author{
(Received January 10, 2012)
}

\author{
Takumi AkiYama*, Wakana Sekiguchi, Takeshi Yamazaki and Hiroshi AkiYama \\ National Institute of Health Sciences: \\ 1-18-1 Kamiyoga, Setagaya-ku, Tokyo 158-8501, Japan; \\ * Corresponding author
}

\begin{abstract}
Enzymatically hydrolyzed guar gum (EHGG), which is used as a thickener or a soluble dietary fiber, is produced by partial hydrolysis of the guar gum (GG) backbone using mannan endo$\beta$-1,4-mannosidase. In this study, we compared and evaluated 3 methods to distinguish EHGG from other polysaccharides used as food additives or monosaccharides. The first method is based on cross-linking reaction of saccharide hydroxyl groups mediated by borate ions. EHGG showed gelation and was distinguished from some soluble polysaccharides, which did not form gels, and also from polysaccharides with low solubility in water. The second method is based on co-gelation with xanthan gum. It was applicable to GG, but not to EHGG. The third method is based on the alcohol precipitation of hydrophilic polymers. EHGG, some soluble polysaccharides and monosaccharides were dissolved in water at the concentration of $10 \%$, while GG and some polysaccharides were not. The $10 \%$ solutions thus obtained were mixed with 2-propanol at the ratio of $1: 1(\mathrm{v} / \mathrm{v})$. A white precipitate was formed in the EHGG solutions and the tested soluble polysaccharide solutions, while it was not produced in the monosaccharide solutions. This result demonstrated that soluble polysaccharides including EHGG can be distinguished from polysaccharides with low solubility or monosaccharides by the third method.
\end{abstract}

Key words: enzymatically hydrolyzed guar gum; polysaccharide; sodium borate; alcohol precipitation

\section{Introduction}

Guar gum (GG) is a galactomannan extracted from ground endosperm of guar beans. The basic structure of $\mathrm{GG}^{1)}$ (Fig. 1) is composed of a linear backbone chain of $\beta$-1,4-linked mannose residues to which galactose residues are $\alpha-1,6$-linked at every second mannose. The ratio of mannose:galactose in GG is about $2: 1$ and the average molecular weight is approximately $200,000 \mathrm{Da}^{1)}$.

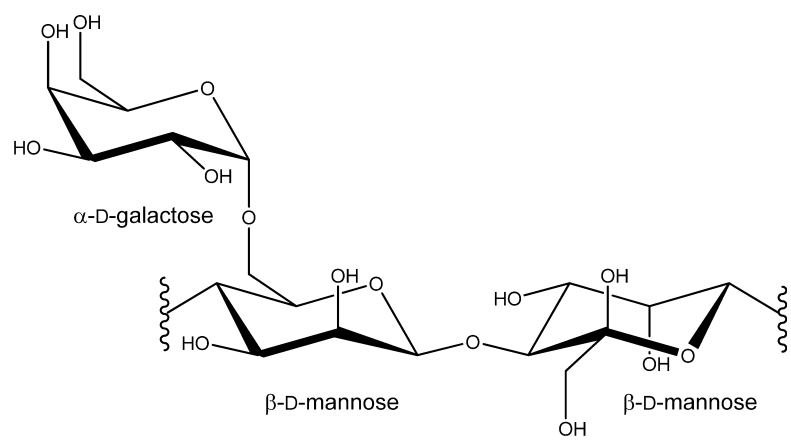

Fig. 1. Partial structure of guar gum (GG) and enzymatically hydrolyzed guar gum (EHGG)
Enzymatically hydrolyzed guar gum (EHGG), which is also called partially hydrolyzed guar gum (PHGG), is produced by partial hydrolysis of the GG backbone with mannan endo- $\beta$-1,4-mannosidase. The average molecular weight of EHGG is approximately $20,000 \mathrm{Da}^{2,3)}$ EHGG uptake has been shown to suppress postprandial serum lipid levels after consumption of a meal high in fat and cholesterol, reducing the absorption of fat and cholesterol through the depletion flocculation mecha$\operatorname{nism}^{4,5)}$. In addition, EHGG uptake decreased the symptoms in constipation-predominant and diarrhea-predominant forms of irritable bowel syndrome, as well as decreasing abdominal pain ${ }^{6}$. It was reported that diarrhea caused by ingestion of sugar alcohol sweeteners is suppressed by EHGG uptake ${ }^{7)}$.

EHGG is included in the List of Existing Food Additives in Japan*1 and is classified as a thickener. However, the specification as food additive has not been established by the Ministry of Health, Labour and Welfare of

*1 Notification No. 120 (Apr. 16, 1996), the Ministry of Health, Labour and Welfare of Japan.

*2 Notice No. 0701007 (Jul. 1, 2005), Department of Food Safety, Pharmaceutical and Food Safety Bureau, the Ministry of Health, Labour and Welfare of Japan.

* akiyamat@nihs.go.jp 
Japan. Specifications for EHGG as a food additive have not been defined in any country. Therefore, we have been investigating the proposal specification for Japan's Specifications and Standards for Food Additives, including identification tests.

Tests for identification, which can distinguish EHGG from other food additives, are necessary for standardization, and to establish specifications and safety requirements for EHGG. The self-regulatory specification of the Japan Food Additives Association (JFAA) has 2 identification tests for EHGG based on conventional methods for gums ${ }^{8)}$. EHGG as a soluble dietary fiber is specified as a standardized food for specialized health uses (standardized FOSHU) in Japan*2. Identification tests in this specification are similar to those in the specification of JFAA. To our knowledge, there are no reports on EHGG identification tests in scientific publications.

According to the JECFA specifications for gums, the analysis of individual monosaccharides in gums is recommended for identification tests. We previously developed an identification method for gums by analyzing the diethyldithioacetal derivatives of monosaccharides ${ }^{9)}$. However, we considered that this test would be inappropriate as an identification test for routine inspection due to its complexity and time-consuming nature. In the present paper, we compared and evaluated 3 simple methods for use as identification tests for EHGG.

\section{Materials and Methods}

\section{Samples and reagents}

Gum samples of EHGG, GG and xanthan gum were kindly provided by JFAA. Only 3 EHGG products from 2 manufacturers are circulated by JFAA member companies. Sample A was a product from one manufacturer and samples $\mathrm{B}$ and $\mathrm{C}$ were from the other.

Other gums were of reagent grade. Pectin from apple, pectin from citrus, swelling alginic acid, non-swelling alginic acid, $\kappa$-carrageenan, $\lambda$-carrageenan and dextran (molecular weight: 32,000-40,000) were purchased from Wako Pure Chemical Industries, Ltd. Gum arabic from Acacia senegal and gum tragacanth were products of Sigma. Gum ghatti and pullulan were purchased from MP Biochemicals and Hayashibara, respectively.

D-Galactose, D-mannose, sodium borate and 2-propanol were of reagent grade, purchased from Wako Pure Chemical Industries, Ltd. Ultrapure water $(>18 \mathrm{M} \Omega \mathrm{cm})$, prepared with a Milli-Q SP Reagent Water System (Millipore, Billerica, U.S.A.), was used throughout the study.

\section{The method based on cross-linking reaction with borate ions}

Two grams of gum sample was moistened with $0.4 \mathrm{~mL}$ of 2-propanol. Ten milliliters of water was added gently, and the mixture was mixed vigorously until the gum was completely dispersed. Ten milliliters of $5 \%$ sodium borate solution was added while mixing gently.

\section{The method based on co-gelation with xanthan gum}

Approximately 1, 2 or $4 \mathrm{~g}$ of sample was mixed with approximately 1, 2 or $4 \mathrm{~g}$ of xanthan gum, and $4 \mathrm{~mL}$ of 2-propanol was then added. Two hundred milliliters of water was gradually added with vigorous mixing until the gum was completely dispersed. Approximately half of this solution was transferred to another vessel, heated at $95^{\circ} \mathrm{C}$ for 10 minutes and then cooled in an ice-water bath.

\section{The method based on precipitation using 2-propanol}

Two hundred milligrams of gum sample was transferred to a glass tube and $200 \mu \mathrm{L}$ of 2 -propanol was added. Two milliliters of water was added and mixed well. The 10\% mixed monosaccharide solution was prepared by dissolving $74 \mathrm{mg}$ of D-galactose and $148 \mathrm{mg}$ of D-mannose in $2 \mathrm{~mL}$ of water. Two milliliters of 2-propanol was added and vigorously mixed.

\section{Results and Discussion}

EHGG has high solubility in water because its molecular weight is about one-tenth of that of GG. This property may be utilized to distinguish EHGG from insoluble polysaccharides. On the other hand, its structure as a galactomannan and high molecular weight are useful to distinguish EHGG from some polysaccharides and monosaccharides. In the present paper, we compared and evaluated 3 methods, which are based on different principles, for distinguishing EHGG from polysaccharides used as food additives and monosaccharides.

The applicability of a method based on cross-linking reaction with borate ions (the cross-linking reaction method)

A method based on cross-linking reaction of saccharides via hydroxy group mediated by borate ions was tested. Polyvinyl alcohol, galactomannans and glucomannans are known to crosslink with borate ions. A method based on this property is utilized for the identification test of galactomannans GG and locust bean gum in Japan's Specifications and Standards for Food Additives $^{10)}$. In this test, $2 \mathrm{~g}$ of a sample is moistened with $4 \mathrm{~mL}$ of 2-propanol and then dissolved in $200 \mathrm{~mL}$ of water. A 10-mL aliquot of each sample solution, which contains $0.1 \mathrm{~g}$ of a sample, is mixed with $2 \mathrm{~mL}$ of $5 \%$ sodium borate solution. A positive result in this test is judged by the formation of a gel or a significant increase in viscosity. A method based on cross-linking reaction with borate ions is adopted by the identification tests for EHGG in the self-regulatory specifications of $\mathrm{JFAA}^{8)}$ and in the specification as a standardized FOSHU. Twenty grams of a sample is used in these tests.

The applicability of the method based on cross-linking reaction was assessed using all three products circulated by JFAA member companies. In the assessed method, the concentration of a sample and sodium borate were twelve-fold and three-fold higher, respectively, than those in the test for GG because EHGG didn't form a gel in the test for GG. The heating step in the test for GG, which is used to distinguish GG from locust bean gum, 
Table 1. Applicability of the cross linking reaction method and the alcohol precipitation method

\begin{tabular}{lccc}
\hline \hline & \multicolumn{2}{c}{ Cross linking method } & \multicolumn{2}{c}{ Alcohol precipitation method } \\
\cline { 2 - 3 } & Solubility in water & Gelation & Solubitily in water \\
\cline { 2 - 3 } Galactose+mannose & NT & PT & Dissolved \\
EHGG & Dissolved & Yes & Dissolved \\
Gum arabic & Dissolved & No & Dissolved \\
Dextran & Dissolved & No & Dissolved \\
Pullulan & Dissolved & No & Dissolved \\
GG & Lump & NT & Paste \\
Pectin & Paste & YT & Yes \\
Gum ghatti & Lump & NT \\
Xanthan gum & Lump & NT & Dense suspension \\
Gum tragacanth & Lump & NT & Pense suspension \\
Swelling alginic acid & Lump & NT & Paste \\
Non-swelling alginic acid & Paste & NT & Paste \\
$\kappa$-Carrageenan & Lump & NT & Precipitation \\
$\lambda$-Carrageenan & Lump & NT & Precipitation \\
\hline
\end{tabular}

NT: Not tested.

was omitted. Two grams of a sample was moistened with $0.4 \mathrm{~mL}$ of 2-propanol and then $10 \mathrm{~mL}$ of water was added. Vigorous mixing of the solution completely dissolved samples A and C. Sample B gave a suspension with low viscosity, indicating that the majority of the powder was dissolved. When $10 \mathrm{~mL}$ of $5 \%$ sodium borate solution was added to the solution, we confirmed that a gel was immediately formed with all samples. Increased viscosity indicated that EHGG has sufficient molecular weight and suitable structure to aggregate when combined with borate.

The applicability of this method was assessed with various polysaccharides used as food additives. To pass the test, a polysaccharide must be dissolved or suspended in water and form a gel when borate solution is added. Out of 36 thickening polysaccharides included in the List of Existing Food Additives in Japan, GG, pectin, gum arabic, gum ghatti, gum tragacanth, alginic acid, $\kappa$-carrageenan, $\lambda$-carrageenan, dextran and pullulan were obtained. First, $2 \mathrm{~g}$ of gum was moistened with $0.4 \mathrm{~mL}$ of 2-propanol and then $10 \mathrm{~mL}$ of water was added. After vigorous mixing, gum arabic, dextran and pullulan were dissolved. Other gums form lumps or a paste (Table 1). Next, $10 \mathrm{~mL}$ of $5 \%$ sodium borate solution was added to the solution of gum arabic, dextran and pullulan. Gel was not formed for any sample. There is no report indicating that arabinogalactans or glucans crosslink with borate. These results demonstrate that EHGG is distinguished from polysaccharides with low solubility in water and also from some soluble polysaccharides in this test.

The applicability of a method based on co-gelation with xanthan gum (the co-gelation method)

We next evaluated the applicability of a method based on co-gelation with xanthan gum. A galatomannan generally forms a gel when it is mixed with polysaccharides composed of glucuronic acid, e.g., xanthan gum. This gelation is called co-gelation or viscosity synergy. An iden- tification test in the self-regulatory specifications of JFAA adopts this principle. We examined whether EHGG would induce co-gelation when mixed with xanthan gum. An aliquot of EHGG (sample C) was mixed with xanthan gum. Tested combinations of EHGG and xanthan gum were as follows: (weight of EHGG: weight of xanthan gum $)=(1 \mathrm{~g}: 1 \mathrm{~g}), \quad(2 \mathrm{~g}: 1 \mathrm{~g}), \quad(2 \mathrm{~g}: 2 \mathrm{~g})$, $(4 \mathrm{~g}: 1 \mathrm{~g}),(4 \mathrm{~g}: 4 \mathrm{~g})$. Next, $4 \mathrm{~mL}$ of 2 -propanol was added, and the mixture was dispersed in $200 \mathrm{~mL}$ of water. Approximately half of each mixture was heated at $95^{\circ} \mathrm{C}$ and then cooled in an ice-water bath. Significant increases in viscosity or gelation were not observed in any of the tested combinations. In contrast, under identical test conditions, the mixing of $1 \mathrm{~g}$ GG sample and $1 \mathrm{~g}$ xanthan gum resulted in gel formation. Considering this result, it is speculated that the molecular length of EHGG was too small to form an insoluble complex with xanthan gum. EHGG is not distinguished from monosaccharides by this method.

The applicability of a method based on precipitation using 2-propanol (the alcohol precipitation method)

A method based on the alcohol precipitation of hydrophilic polymers was also evaluated. This property of hydrophilic polymers had been utilized in some purification procedures, i.e., the precipitation of plasmid DNA in $50 \%$ 2-propanol or in $70 \%$ to $80 \%$ ethanol. We examined the applicability of a method based on this property for EHGG identification. Two hundred milligrams of GG or EHGG (sample A) was placed in a glass tube, moistened with $200 \mu \mathrm{L}$ of 2-propanol, and then dissolved in $2 \mathrm{~mL}$ of water to make $10 \%$ solution. EHGG was completely dissolved in water, while GG resulted in a turbid solution. After $2 \mathrm{~mL}$ of 2-propanol was added, a white precipitate was observed. A mixed monosaccharide solution that contains the same ratio and concentration as $10 \%$ EHGG solution was tested. A solution containing 3.7\% galactose and $7.4 \%$ mannose did not produce a white precipitate or any insoluble material upon addition of 


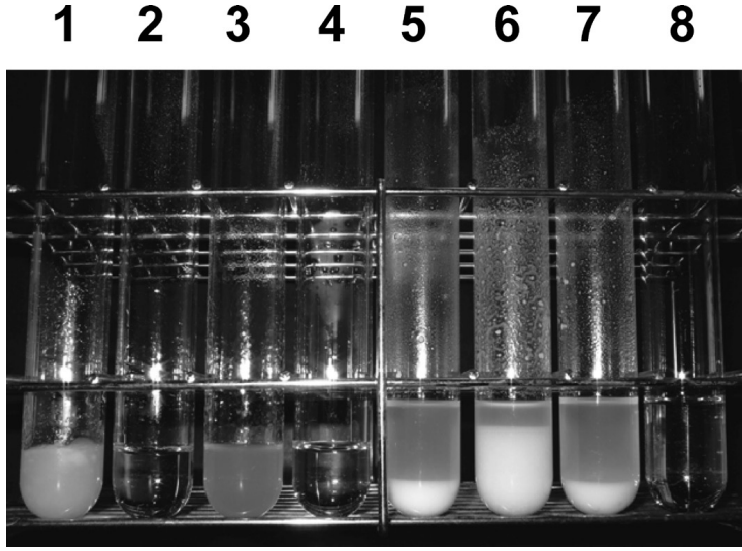

Fig. 2. Precipitation of enzymatically hydrolyzed guar gum (EHGG) in the alcohol precipitation method

1 to 4: without 2-propanol; 1, 10\% guar gum; 2 to 4, 10\% EHGG (samples A, B and C from the left). 5 to 8: after 2-propanol addition; 5 to $7,10 \%$ EHGG (samples A, B and C from the left); 8, mixed monosaccharide solution containing 3.7\% galactose and $7.4 \%$ mannose

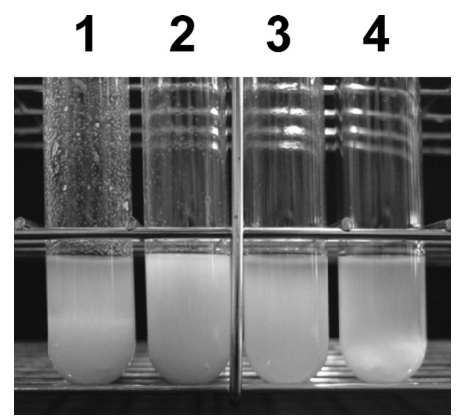

Fig. 3. Precipitation of gums in the alcohol precipitation method

1, $10 \%$ EHGG (sample C); 2, $10 \%$ gum arabic from Acacia senegal; 3, 10\% dextran; 4, 10\% pullulan

2-propanol. In addition, with this method, the other two products tested (sample B and sample C) produced white precipitates (Fig. 2). Although sample B did not dissolve completely in water (tube 3 in Fig. 2), precipitation (tube 6 in Fig. 2) was clearly distinguishable. These results demonstrate that $\mathrm{EHGG}$, which is a polymer of 100 to 150 sugars, can be distinguished from GG based on solubility in water and also from monosaccharides based on precipitation in 50\% 2-propanol using this proposed method.

Other gums, pectin, gum arabic, gum ghatti, gum tragacanth, alginic acid, $\kappa$-carrageenan, $\lambda$-carrageenan, dextran and pullulan, were tested using this method. Gum arabic, dextran and pullulan were dissolved completely. When $2 \mathrm{~mL}$ of 2-propanol was added, a white precipitate was observed for each of these samples (Fig. 3 and Table 1).
It was demonstrated that the alcohol precipitation method was able to distinguish EHGG from GG, polysaccharides with low solubility and monosaccharides.

\section{Conclusion}

1) EHGG is distinguished from polysaccharides with low solubility in water and also from some soluble polysaccharides by the cross-linking reaction method.

2) EHGG is not distinguished from monosaccharides by the co-gelation method.

3) The alcohol precipitation method was able to distinguish soluble polysaccharides including EHGG from polysaccharides with low solubility and monosaccharides.

\section{References}

1) Baker, C. W., Whistler, R. L. Distribution of D-galactosyl groups in guaran and locust-bean gum. Carbohydr. Res., 45, 237-243 (1975).

2) Yamamoto, T., Yamamoto, S., Miyahara, I., Matsumura, Y., Hirata, A., Kim, M. Isolation of $\beta$-mannan hydrolyzing enzyme and hydrolysis of guar gum by the enzyme isolated. Denpun Kagaku, 37, 99-105 (1990).

3) Takahashi, H., Wako, N., Okubo, T., Ishihara, N., Yamanaka, J., Yamamoto, T. Influence of partially hydrolyzed guar gum on constipation in women. J. Nutr. Sci. Vitaminol., 40, 251-259 (1994).

4) Kondo, S., Xiao, J. Z., Takahashi, N., Miyaji, K., Iwatsuki, K. Suppressive effects of dietary fiber in yogurt on the postprandial serum lipid levels in healthy adult male volunteers. Biosci. Biotechnol. Biochem., 68, 1135-1138 (2004).

5) Minekus, M., Jelier, M., Xiao, J. Z., Kondo, S., Iwatsuki, K., Kokubo, S., Bos, M., Dunnewind, B., Havenaar, R. Effect of partially hydrolyzed guar gum (PHGG) on the bioaccessibility of fat and cholesterol. Biosci. Biotechnol. Biochem., 69, 932-938 (2005).

6) Parisi, G. C., Zilli, M., Miani, M. P., Carrara, M., Bottona, E., Verdianelli, G., Battaglia, G., Desideri, S., Faedo, A., Marzolino, C., Tonon, A., Ermani, M., Leandro, G. High-fiber diet supplementation in patients with irritable bowel syndrome (IBS): a multicenter, randomized, open trial comparison between wheat bran diet and partially hydrolyzed guar gum (PHGG). Dig. Dis. Sci., 47, 1697-1704 (2002).

7) Nakamura S., Hongo R., Moji K., Oku T. Suppressive effect of partially hydrolyzed guar gum on transitory diarrhea induced by ingestion of maltitol and lactitol in healthy humans. Eur. J. Clin. Nutr., 61, 1086-1093 (2007).

8) Japan Food Additives Association ed. Kizon Tenkabutsu Jishu Kikaku, 4th ed. Tokyo, Japan Food Additives Association, 2008, p. 318.

9) Akiyama, T., Yamazaki, T., Tanamoto, K. Analysis of thickening polysaccharides by the improved diethyldithioacetal derivatization method, Shokuhin Eiseigaku Zasshi (Food Hyg. Saf. Sci.), 52, 40-46 (2011).

10) Ministry of Health, Labour and Welfare of Japan. Shokuhin Tenkabutsu Koteisho (Japan's Specifications and Standards for Food Additives) 8th ed., 2007. 